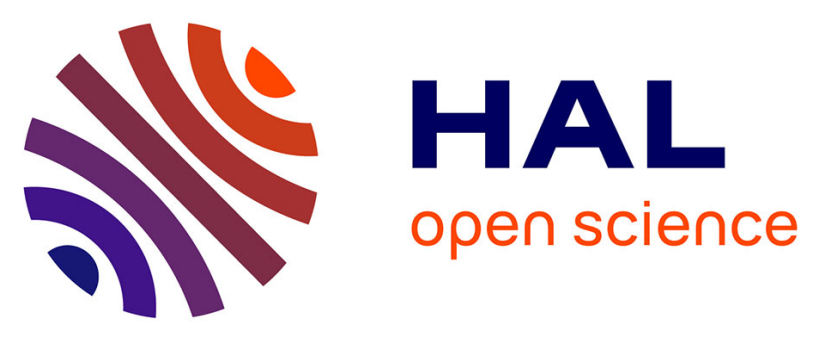

\title{
Chronic administration of minoxidil protects elastic fibers and stimulates their neosynthesis with improvement of the aorta mechanics in mice
}

Wassim Fhayli, Marjorie Boyer, Zeinab Ghandour, Marie-Paule Jacob, Jean-Pierre Andrieu, Barry Starcher, Eric Estève, Gilles Faury

\section{To cite this version:}

Wassim Fhayli, Marjorie Boyer, Zeinab Ghandour, Marie-Paule Jacob, Jean-Pierre Andrieu, et al.. Chronic administration of minoxidil protects elastic fibers and stimulates their neosynthesis with improvement of the aorta mechanics in mice. Cellular Signalling, 2019, 62, pp.109333. 10.1016/j.cellsig.2019.05.018 . hal-02271542

HAL Id: hal-02271542

https://hal.univ-grenoble-alpes.fr/hal-02271542

Submitted on 21 Dec 2021

HAL is a multi-disciplinary open access archive for the deposit and dissemination of scientific research documents, whether they are published or not. The documents may come from teaching and research institutions in France or abroad, or from public or private research centers.
L'archive ouverte pluridisciplinaire HAL, est destinée au dépôt et à la diffusion de documents scientifiques de niveau recherche, publiés ou non, émanant des établissements d'enseignement et de recherche français ou étrangers, des laboratoires publics ou privés.

\section{(ㄷ)(1) $\$$}

Distributed under a Creative Commons Attribution - NonCommerciall 4.0 International 


\title{
Molecular dynamics studies on the aggregating behaviors of cellulose
}

$$
\text { molecules in } \mathrm{NaOH} / \text { urea aqueous solution }
$$

\author{
Gang Liu ${ }^{\mathrm{a}}$, Wei Li ${ }^{\mathrm{b}}$, Liangliang Chen ${ }^{\mathrm{c}}$, Xin Zhang ${ }^{\mathrm{a}}$, Dechuang Niu', Yuyan Chen ${ }^{\mathrm{a}}$,
} Shiling Yuan ${ }^{\mathrm{d}}$, Yiling Bei ${ }^{\mathrm{a}}$, Qingzeng Zhu,

${ }^{a}$ Key Laboratory of Special Functional Aggregated Materials, Ministry of Education, School of Chemistry and Chemical Engineering, Shandong University, Jinan 250199, P. R. China

${ }^{\mathrm{b}}$ School of Chemistry and Chemical Engineering, Qiannan Normal University for Nationalities, Duyun, Guizhou, 558000, P. R. China

${ }^{\mathrm{c}}$ National Engineering Laboratory for Surface Transportation Weather Impacts Prevention, Broadvision Engineering Consultants, Kunming 650041, China

${ }^{\mathrm{d}}$ Key Laboratory of Special Functional Aggregated Materials, School of Chemistry and Chemical Engineering, Shandong University, Jinan 250199, P. R. China

Abstract Aggregating behaviors of cellulose molecules in $\mathrm{NaOH} /$ urea aqueous solution were investigated using a molecular dynamics (MD) simulation method. All the components, including cellulose, $\mathrm{NaOH}$, urea and $\mathrm{H}_{2} \mathrm{O}$ were combined into the simulation. By monitoring the transformation of cellulose aggregates, we quantitatively revealed the solvent aggregating behaviors around cellulose molecules in the whole simulation process. And the aggregation evolution of solvent components around cellulose molecules, distributions of the solvent components in cellulose ICs, and the impacts of urea and $\mathrm{NaOH}$ in the dissolving process were discussed. In the simulation, the aggregated structure of cellulose molecules was dramatically changed and went through a dispersion process, equilibrium process and reaggregation process. The dramatic transformation of the cellulose aggregated structure indicates that the solvent components prefer to penetrate into the cellulose molecular sheets from the direction perpendicular to the molecular sheet. In the dispersion process, sodium ions around O6 and hydroxide ions around $\mathrm{O} 3$ and $\mathrm{O} 2$ play crucial roles in the dissolution of cellulose. In the reaggregation process, the sodium ions near O6 and the urea around acetalic oxygen atoms impact the stability of cellulose inclusion complexes, and obvious decrements in these solvent components were observed.

\footnotetext{
* Corresponding author address: Key Laboratory of Special Functional Aggregated Materials, Ministry of Education, School of Chemistry and Chemical Engineering, Shandong University, Jinan 250199, PR China. E-mail address: qzzhu@sdu.edu.cn
} 
Keywords MD simulation; cellulose; dissolution; $\mathrm{NaOH}$ /urea aqueous solution

\section{Introduction}

Cellulose, a major structural component of plant cell walls, is one of the most abundant natural polymers on earth. Due to its biodegradability and renewability, cellulose has been utilized and exploited worldwide [1-3]. However, the strong interactions between cellulose chains, especially in the crystalline cellulose region where the long cellulose chains are tightly stacked together sheet by sheet, make native cellulose hard to infiltrate and dissolve in common solvents [4-7]. This recalcitrance to dissolve has long been a key challenge for the further application of cellulose.

Studies of highly efficient solvent systems for cellulose have been widely carried out and a series of outstanding solvent systems has been developed, such as $\mathrm{N}$ methylmorpholine-N-oxide [8], lithium chloride/N,N-dimethylacetamide [9], ionic liquids [10], inorganic molten salt hydrates [11] and so on. Among these established solvent systems, the alkali/urea or alkali/thiourea aqueous solution has received considerable attention and is a promising solvent system for the dissolution of cellulose. Due to its simplicity, low cost, low toxicity, environmental friendliness and ease of recovery, this kind of solvent system has attracted much attention. Based on in-depth studies of mercerization and the viscose process, the dissolution of cellulose was first found at certain $\mathrm{NaOH}$ concentrations [12-13]. However, for native cellulose, the effective dissolution only occurred in cellulose with a low degree of polymerization. It was also found that additives could improve the dissolution [14]. However, alkali solutions with additives such as urea or thiourea did not attracted attention until the 1990s. In 1990, Laszkiewicz demonstrated that urea or thiourea could significantly improve cellulose solubility in alkali solution systems [15-17]. Zhang's group has also studied the dissolution of cellulose in alkali/urea or alkali/thiourea aqueous systems. And a series of alkali/urea or alkali/thiourea aqueous systems with quick dissolving power have been developed [18-21]. These studies reported that the efficient dissolution of cellulose could be achieved in precooled $7 \mathrm{wt} \% \mathrm{NaOH} / 12 \mathrm{wt} \%$ urea [22], $4.6 \mathrm{wt} \%$ $\mathrm{LiOH} / 15 \mathrm{wt} \%$ urea [23] or $9.5 \mathrm{wt} \% \mathrm{NaOH} / 4.5 \mathrm{wt} \%$ thiourea [24] within several minutes. This kind of solutions is a very valid dissolution system for native cellulose. 
Some experimental studies have been done to reveal the dissolution mechanism of cellulose in $\mathrm{NaOH} /$ urea aqueous solution. Zhang and his coworkers reported that $\mathrm{Na}^{+}$ has a direct interaction with cellulose and urea molecules, and proposed a model based on cellulose inclusion complexes (ICs) [25-26]. They proposed that $\mathrm{Na}^{+}$occupies the area of the hydroxyl groups of cellulose and plays a crucial role in breaking the hydrogen bonds within cellulose chains. The urea is bonded to $\mathrm{NaOH}$ hydrates on the surface of ICs and prevents the aggregation of cellulose chains [27]. The MD simulation method has also been used to study the dissolving process of cellulose, Miyamoto found the dense areas of $\mathrm{Na}^{+}$between $\mathrm{O} 2$ and $\mathrm{O} 3$ of cellulose and claimed that $\mathrm{Na}^{+}$penetrated into cellulose sheets promoting the separation of cellulose chains [28]. We have also described the detailed structure of cellulose ICs around a single cellulose chain using the molecular dynamics method [29].

Although the studies mentioned above provide useful knowledge for the dissolving mechanism of cellulose, there remains much controversy regarding the impact of alkali and urea in the dissolving process of cellulose, for example. Many researchers, such as Nishimura and Takahashi, agree that $\mathrm{Na}^{+}$breaks the intermolecular hydrogen bonds within cellulose chains [30-31]. However, Xiong indicated that the $\mathrm{OH}^{-}$breaks the hydrogen bonds and that $\mathrm{Na}^{+}$hydration stabilizes the hydrophilic hydroxyl groups [32]. For the interaction between urea and cellulose, Egal and Isobe concluded that urea does not interact with either $\mathrm{NaOH}$ or cellulose [33-34]. However, Cai and Lund believe that the urea adsorbs onto the surface of the cellulose and the hydrogen bonding between urea and cellulose directly attenuates the hydrophobic effect of the cellulose chain [3536]. We have also confirmed that urea molecules can form hydrogen bonds with the hydroxylic and acetalic oxygen atoms of cellulose [29]. In addition, some details remain unclear regarding the dissolving process of cellulose, such as how each of the solvent components behaves in the cellulose dissolution process, how the cellulose/ $\mathrm{NaOH} / \mathrm{urea}$ solution becomes a gel if the temperature increases, and what the detailed structure of the cellulose ICs is, and so on.

In this study, to gain deeper insights into the atomic details of cellulose dissolution, an MD simulation method was employed to investigate the aggregating behavior of 
cellulose molecules in $\mathrm{NaOH} /$ urea aqueous solution. All the components, including cellulose, $\mathrm{NaOH}$, urea and $\mathrm{H}_{2} \mathrm{O}$ were combined into the simulation. Focusing on the complicated interaction between cellulose molecules and solvent particles, we investigated the transformation of cellulose aggregates in $\mathrm{NaOH} / \mathrm{urea}$ aqueous solution. And we quantitatively revealed the aggregating behaviors of solvent particles around cellulose molecules in the whole simulation process. The aggregation evolution of solvent components around cellulose molecules, distributions of the solvent components in cellulose ICs, and the impacts of urea and $\mathrm{NaOH}$ in the dissolving process were also discussed. We expect this work will give a better insight into the interaction between cellulose and solvent components, and promote the design of new cellulose aqueous solution systems.

\section{Computational procedure}

The MD simulation was carried out using the GROMACS free software package (version 2019.2). The cellulose was parameterized by the CHARMM36 carbohydrate force field [37-38]. The urea molecules were modeled using the same parameters used in the report by Weerashinghe and Smith [39]. The sodium ions and hydroxide ions were modeled with CHARMM36 parameters, and water was described by the TIP3P model. A sheet of cellulose with 6 cellulose molecules was extracted from an I $\beta$ crystal structure constructed using a cellulose-builder [40] and the degree of polymerization of the cellulose molecules was 8 .

Initially, a cellulose sheet with 6 cellulose molecules, 524 sodium ions, 524 hydroxide ions, 600 urea and 13437 TIP3P water molecules were randomly dispersed in a cubic box $(8.12 \times 8.12 \times 8.12 \mathrm{~nm})$ with periodic boundary conditions (Fig. 1). The periodic boundary conditions were applied in all directions. The initial configurations were conducted by energy minimization using the steepest descent method. Then, all the simulations were conducted in a normal pressure and temperature (NPT) ensemble for $2 \mathrm{~ns}$ to make the density of the system reach a reasonable value. Finally, a $120 \mathrm{~ns}$ MD simulation was carried out under the canonical ensemble (NVT). The time step was 2.0 fs. The temperature was kept constant at $261 \mathrm{~K}$ by the Berendsen thermostat algorithm [41]. The particle mesh Ewald (PME) method was used to treat the long- 


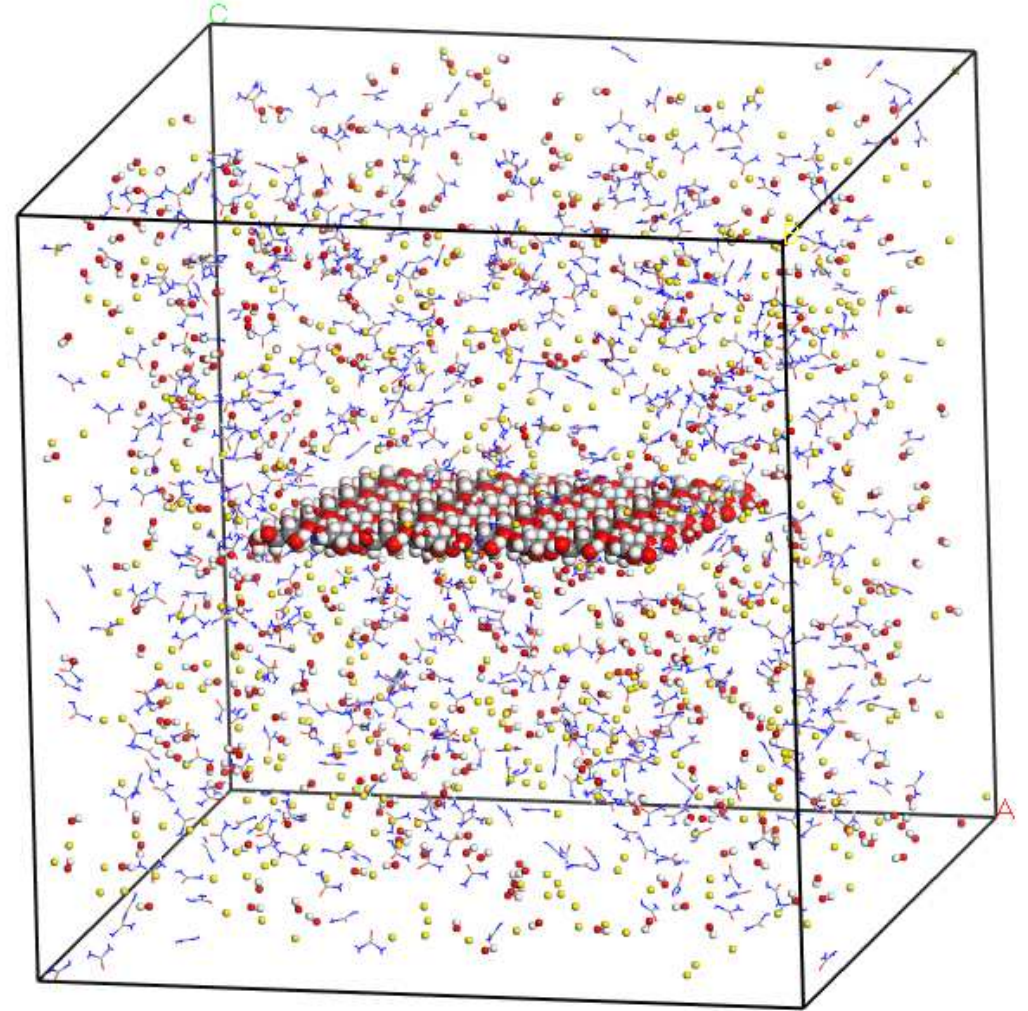

Fig. 1. Setup of the simulation box. The urea molecules are displayed in line mode, sodium ions and hydroxide ions are displayed in ball and stick mode, and the cellulose molecules are displayed in the CPK mode. Colors for the atom scheme are O: red, C: gray, H: white and Na: yellow. The water molecules are not shown for clarity.

\section{Results}

\subsection{Temporal evolution}

MD simulation can reveal the detailed structure at the molecular level and lead to a conclusion for the impact of alkali and urea in the dissolution process of cellulose. In this MD simulation, we were also interested in the possible transformation of the cellulose aggregated structure in $\mathrm{NaOH} /$ urea aqueous solution. As shown in Fig. 2, the aggregation behavior of cellulose molecules was examined. Fig. 2(a) shows the initial structure of cellulose molecules. Fig. 2(b) is the final aggregated structure of cellulose in the NPT simulation. The side-by-side cellulose sheet was rapidly disordered at the initial dissolving stage. A partial offset stacked configuration was obtained at the end of the NPT simulation. Fig. 2(c) shows the aggregated structure of cellulose molecules at $12 \mathrm{~ns}$ of NVT simulation. The ordered offset stacked configurations were formed. 
139 Following the transformation, the cellulose molecules rapidly dispersed into the precooled $\mathrm{NaOH} /$ urea aqueous solution. As shown in Fig. 2(d), two states of cellulose aggregates (a single molecule, bimolecular offset stacked aggregation) were obtained. In the following simulation from Fig. 2(d) to (f), the single molecule and bimolecular offset stacked configurations showed a relatively high stability. Although there were some stable junctions formed between cellulose molecules, no significant aggregating or dispersing actions were observed.

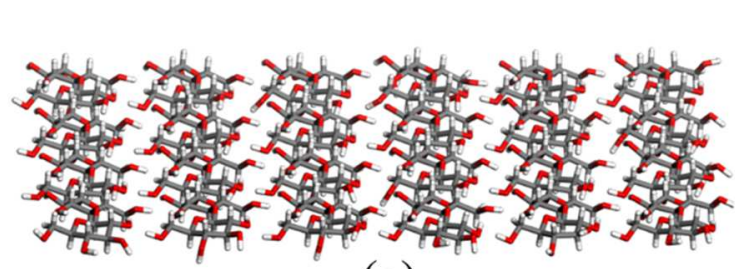

(a)

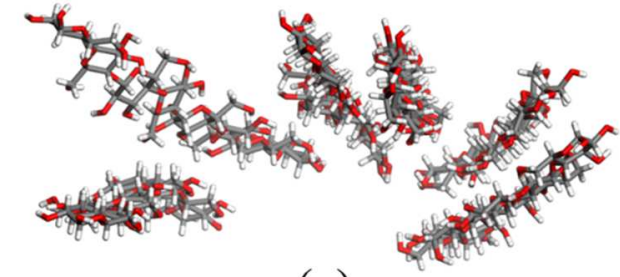

(c)

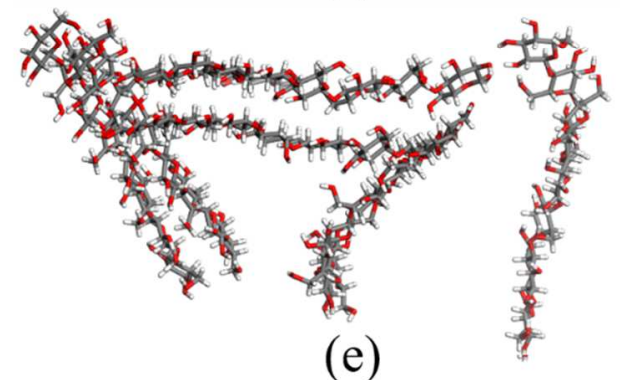

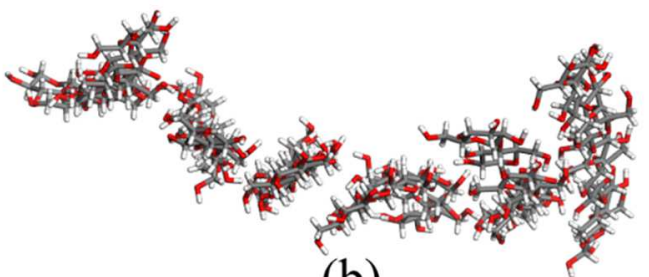

(b)

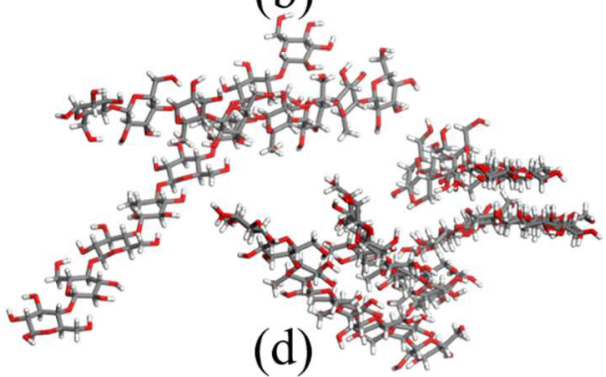

(d)

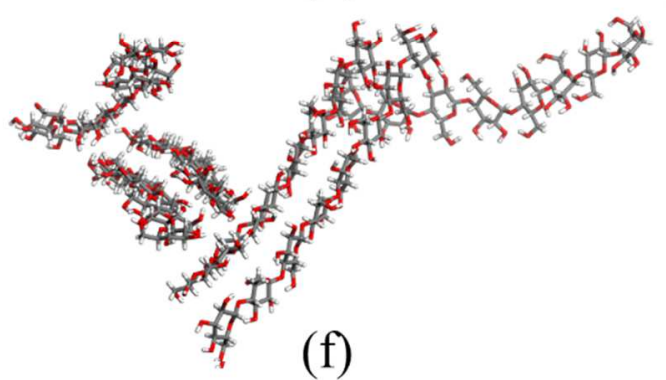

Fig. 2. The evolution of the cellulose aggregated structure in $\mathrm{NaOH} /$ urea aqueous solution. (a) The initial aggregated structure at $0 \mathrm{~ns}$ of the NPT simulation; (b) the final aggregated structure at $2 \mathrm{~ns}$ of the NPT simulation; (c), (d), (e) and (f) show the cellulose aggregated structure for the NVT simulation at $12 \mathrm{~ns}, 28 \mathrm{~ns}, 60 \mathrm{~ns}$ and $102 \mathrm{~ns}$, respectively.

To further confirm the aggregated structure of cellulose molecules in $\mathrm{NaOH} / \mathrm{urea}$ aqueous solution, the solvent accessible surface area (SASA) of cellulose molecules was analyzed. SASA quantitatively represents the contact between cellulose molecules and solvent molecules. It can be used to understand the changes of the aggregated structure of cellulose molecules during the dissolving process. Fig. 3 shows the SASA profile. The red arrows are used to mark the aggregated morphologies of cellulose 
molecules in Fig. 2. A significant fluctuation of SASA is observed in the NPT simulation and the initial period of NVT simulation. This is consistent with the transformation from side-by-side configuration to the offset stacked configuration of cellulose molecules. At about 3 ns, most of cellulose molecules stacked together and the SASA of the cellulose molecules decreased rapidly. At approximately $20 \mathrm{~ns}$, the SASA of the cellulose molecules increases rapidly. This means that the ordered offset stacked aggregates of cellulose rapidly disperse into the $\mathrm{NaOH} /$ urea aqueous solution. After 20 ns, as marked by the red dashed line in Fig. 3, the fluctuation of SASA in the period from $20 \mathrm{~ns}$ to $70 \mathrm{~ns}$ is small and the average value of SASA is approximately $63.64 \mathrm{~nm}^{2}$. The fluctuation of SASA within a narrow range indicates that the dispersed structures of the cellulose molecules are in equilibrium. If the 6 cellulose molecules completely transformed into single molecular dispersion structures, the value of SASA would be $80.88 \mathrm{~nm}^{2}$. The relatively smaller SASA obtained in the MD simulation also suggests that the cellulose molecules are partially aggregated in the $\mathrm{NaOH} /$ urea aqueous solution. It is also worth noting that the SASA profile shows a slight decreasing trend from $70 \mathrm{~ns}$ to $120 \mathrm{~ns}$ (marked by the magenta dashed arrow). This indicates that the cellulose molecules have a very slow aggregating trend.

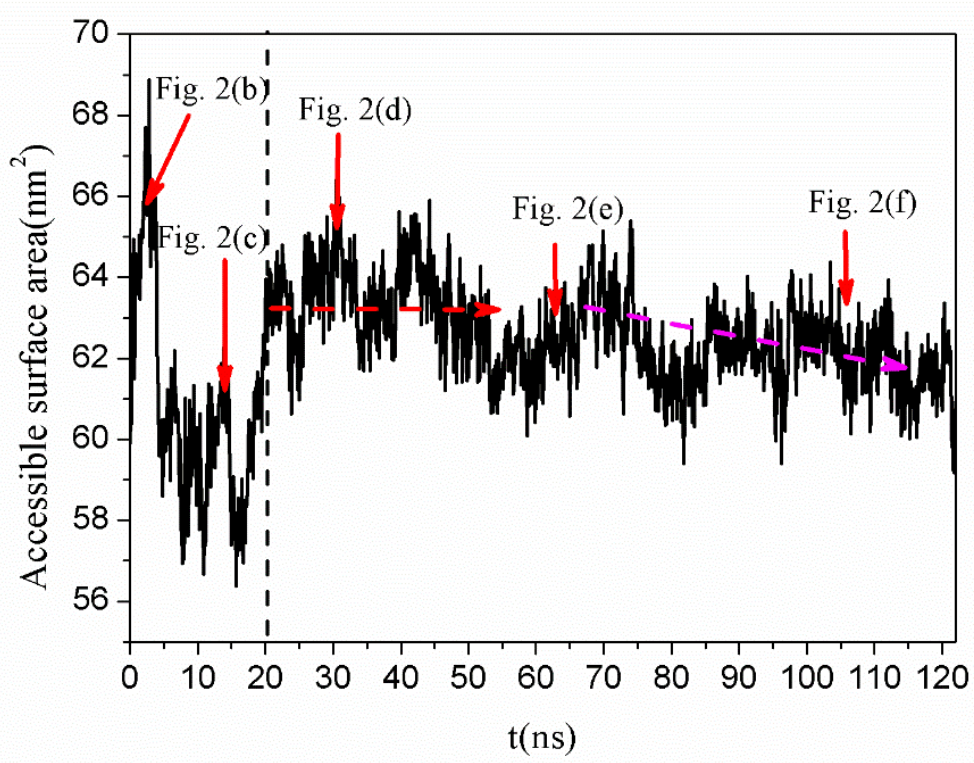

Fig. 3. Solvent accessible surface area profile of cellulose during the simulation.

To analyze whether the rapid dispersion at about $20 \mathrm{~ns}$ is really due to the effect of 
alkali species and urea molecules or just an artifact of the model itself, we conducted additional MD simulations of cellulose in $\mathrm{NaOH}$ aqueous solution and pure water. As shown in Supplementary material Fig. S1, the initial aggregation of cellulose molecules were also found in $\mathrm{NaOH}$ aqueous solution and pure water. That means the initial aggregation of cellulose molecules at $3 \mathrm{~ns}$ may be due to the effect of hydrophobic interaction of cellulose molecules. The subsequent rapid dispersion process at $20 \mathrm{~ns}$ was only found in $\mathrm{NaOH} /$ urea aqueous solution and $\mathrm{NaOH}$ aqueous solution. And the dispersion process in $\mathrm{NaOH} / \mathrm{urea}$ aqueous solution was much earlier than that in $\mathrm{NaOH}$ aqueous solution. This clearly indicates that the dispersion process at about $20 \mathrm{~ns}$ in Fig.3 is mainly due to the effect of alkali species and urea molecules.

During the dissolution process, the aggregated structure of cellulose molecules changed dramatically in the $\mathrm{NaOH} /$ urea aqueous solution. The cellulose molecules rapidly stacked together in the initial stage within $3 \mathrm{~ns}$. The intermolecular interactions within the side-by-side molecular sheet were disrupted and cellulose molecules preferred to offset stack together. With the formation of the offset stacked structure, the cellulose molecules rapidly dispersed into the solutions. An obvious dispersion process was observed at approximately $20 \mathrm{~ns}$. After $20 \mathrm{~ns}$, the stable dispersion configurations for the cellulose molecules, i.e., single molecule and bimolecular offset stacked structure, were formed. In the following simulation, the single molecule and bimolecular offset stacked structure exhibited a relatively high stability. After $70 \mathrm{~ns}$, the cellulose molecules exhibited a slowly reaggregating trend. The transformation from side-by-side molecular sheet to offset stacked structures also gives an insight for the understanding of cellulose crystal dissolution in the $\mathrm{NaOH} /$ urea aqueous solution. It is easy to conclude that the interactions between adjacent cellulose molecules within the side-by-side cellulose sheet are significantly weaker than those in the offset stacked structure. Then, we can predict that the disruption of the cellulose crystalline structures may first occur within the cellulose sheet in the dissolving process.

\subsection{Hydrogen bonds}

It is well known that intermolecular and intramolecular hydrogen bonds play a crucial role in the stability of cellulose. Therefore, the hydrogen bonds within the 
cellulose network should be first broken in the dissolving process, especially for the intramolecular and intermolecular hydrogen bonds. In this work, according to the general hydrogen bonding criteria, i.e., the distance between the donor and the acceptor should be less than $0.35 \mathrm{~nm}$, and the angle is less than 30 degree, the variations of the hydrogen bonds within a cellulose sheet during the NVT simulation were also analyzed to gain deeper insights for the dissolution of cellulose. As shown in Fig. 4, the total number of hydrogen bonds within and between cellulose molecules decreases with the running of the NVT simulation at the initial time. The decrement of hydrogen bonds could be attributed to the disruption of the intermolecular hydrogen bonds. The relative stability of hydrogen bond number from $30 \mathrm{~ns}$ to $60 \mathrm{~ns}$ in the MD simulation illustrates a possible equilibrium for the cellulose dispersion configuration. After $70 \mathrm{~ns}$, the increment in the hydrogen bonds presents the reaggregating process for the cellulose molecules.

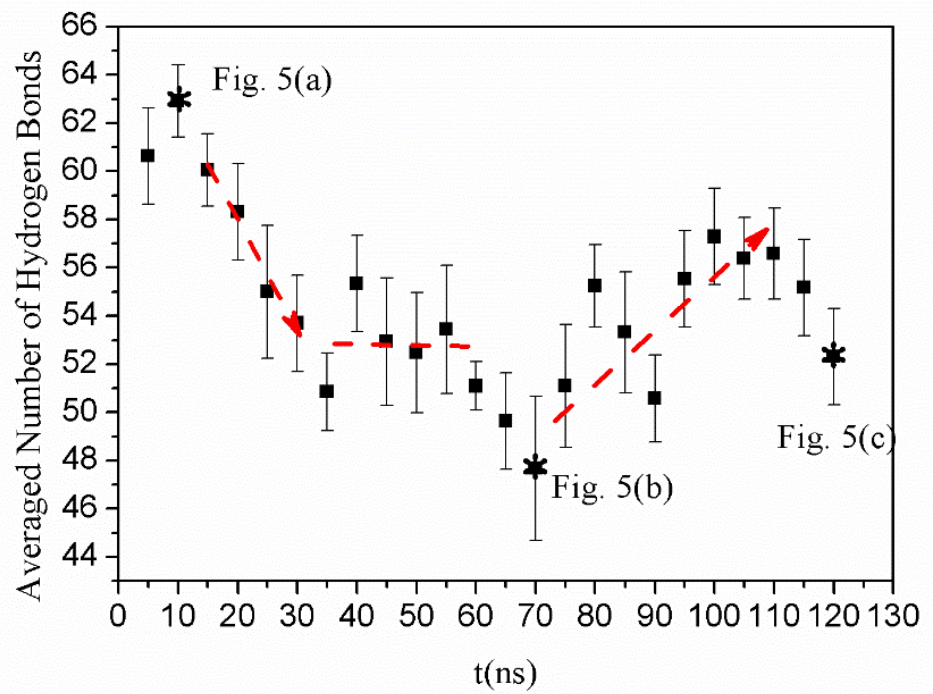

Fig. 4. The average number of hydrogen bonds within and between cellulose molecules over NVT simulation time. Every point was averaged over $5 \mathrm{~ns}$ of MD trajectories.

To further study the relationship between the amount of hydrogen bonds and the cellulose aggregated structure, the hydrogen bonding configurations of cellulose marked with a star in Fig. 4 are shown in Fig. 5. Fig. 5(a) shows the hydrogen bonding structure of cellulose molecules at $12 \mathrm{~ns}$. Due to the stacked configurations of cellulose molecules, many more intermolecular hydrogen bonds were observed. At $70 \mathrm{~ns}$, 